\section{A logarithmic electronic integrator for activity transducers}

\author{
W. T. WOODARD \\ University of South Florida, Tampa, Florida 33620
}

A solid state integrator having an optional logarithmic or linear input/output function, high input impedance, dc input, high sensitivity, low drift, and contact-closure output is described.

The electronic integrator to be described was designed to provide a measure of total activity within a period of time when the input is a voltage analog of activity. While the activity may be of any type for which a suitable transducer is available, the integrator was designed to accept signals from a simple piezoelectric transducer: a paddle-driven crystal phonograph cartridge (Astatic Model 16-U) used to pick up fish-generated activity.

The present device is being used for the same purpose as one described by Bitterman (1966), but provides greater sensitivity and linear or logarithmic input/output functions, utilizes integrated circuits and solid state components, and integrates dc as well as ac inputs.

The basic function performed is one of summing input voltage over time, or, equivalently, computing a running total of the positive area under the curve in a plot of voltage as a function of time. The integrator consists of three basic stages, all direct coupled for de operation: (1) a preamplifier, (2) a voltage-controlled oscillator, and (3) a pulse former and relay driver. These stages are shown in Fig. 1.

The preamplifier provides the basic amplification and high input impedance required by a crystal transducer. An operational amplifier having a high input impedance, a low in put bias current, and a low temperature drift is critical here. Input bias current is compensated by the bias adjustment (R3), and sensitivity (amplification factor) is adjusted by feedback resistor R1. Diode D1, which is responsible for the integrator's logarithmic input/output function, may be replaced with a resistor to change to linear operation The log function is particularly useful when it is desirable to process inputs of a wide voltage range without having to sacrifice sensitivity to low inputs in order to prevent overloading by, and loss of, high inputs.

The voltage-controlled oscillator (Kootsey, 1968) consists of A2 (a less critical amplifier) with a FET (Q1) in the feedback loop, which forms a voltage-controlled variable current source for charging the integrating capacitor C1 (low leakage mylar). When the voltage on $\mathrm{C} 1-$ a voltage proportional to the summed integrator input-reaches a predetermined level, the UJT (Q2) fires, thereby discharging $\mathrm{C} 1$, pulsing $\mathrm{RY1}$, and indicating a unit of activity. For a given input, the output pulse rate is a linear function of $\mathrm{R1}$ (sensitivity control). The integrator may be reset or gated out of operation by unshorting the gate contacts associated with this stage.

The last stage consists of $Q 3$, the relay driver, and an R-C network (R4, C2), which determines the duration of the relay contact closure. The indicated component values give a closure time of about $50 \mathrm{msec}$, but R4 and C2 may be adjusted if other closure times are desired.

While parts layout is not eritical, it is well to keep input and first-stage circuits as far as possible from the

\title{
INTEGRATOR
}

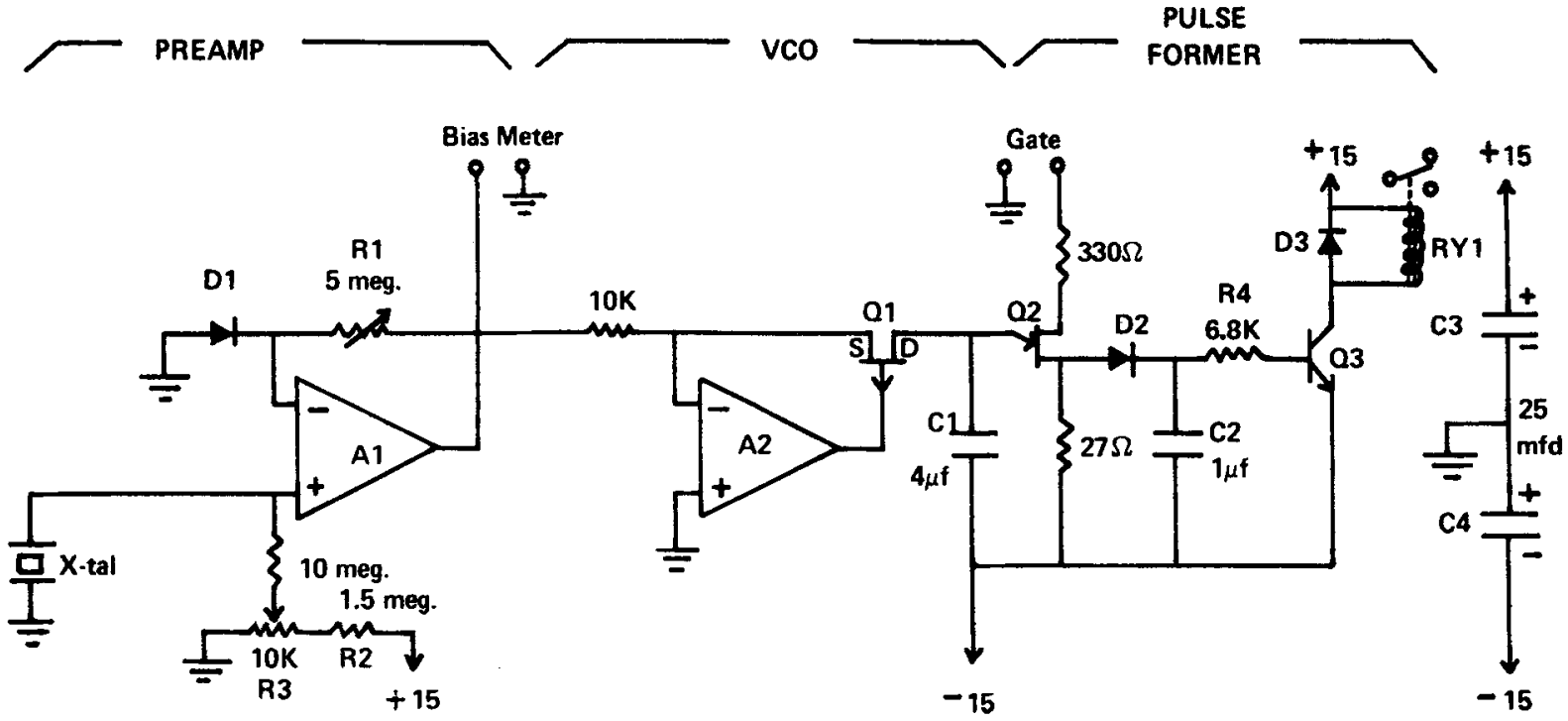

Fig. 1. Integrator circuit. A1, Motorola MC1456G operationalal amplifier, A2, Fairchild $\mu$ A741C op. amp.; D1-3, 1N34 diodes; Q1, HEP803; Q2, HEP310; Q3, HEP55 (any replacement units may be used in place of the noneritical Motorola units listed for Q1-3); R1, linear sensitivity control; R3, 25-turn trimpot bias adjustment; C1, high quality, low leakage mylar integrating capacitor; RY1, Sigma 11 F-6000; C3, C4, needed for power supply isolation when multiple units are operated from a single supply. 

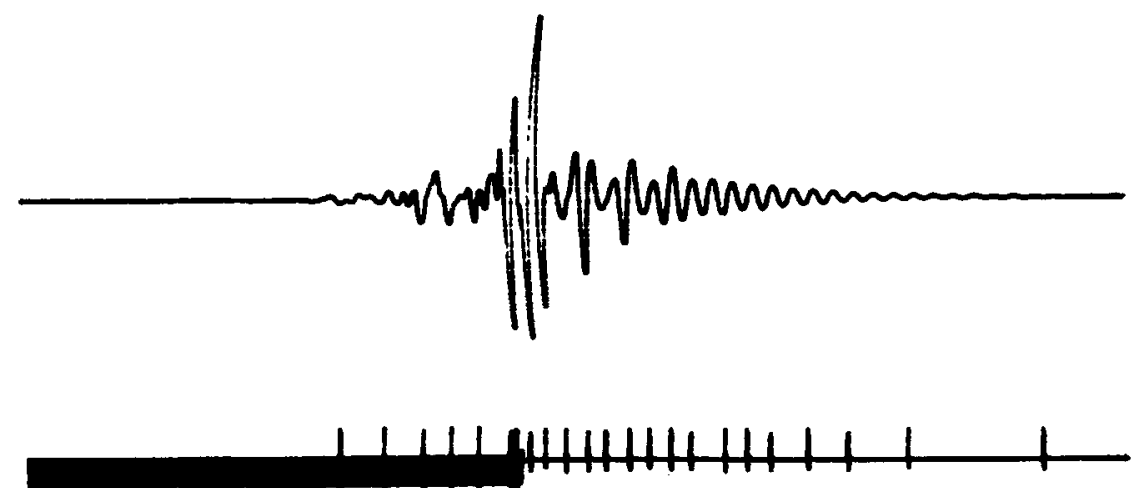

Fig. 2. Sample input/output recording. Upper trace shows the analog activity input, lower trace shows the series of vertical marks generated by the integrator, each representing a unit of activity.

relay and its driver. The present models are constructed on perforated printed-circuit boards, measuring $3 \times 5$ in., and the entire unit is mounted in a $3 \times 5 \times 2$ in. aluminum minibox. The diode, $D 1$, is a critical component and will probably have to be selected from a collection of diodes to yield adequate integrator sensitivity. If multiple integrators must be matched, diodes and input amplifiers must be carefully selected. measured with a dc input varying over two decades.

To illustrate the relationship between the integrator's input and output, Fig. 2 shows a sample recording of goldfish activity in response to a light (CS) and a brief electric shock (US). The fish were being conditioned in the Pavlovian activity chamber described by Bitterman (1966). The thick line at the bottom indicates the CS at the termination of which the US occurred. Superimposed on this line are marks generated by the integrator in response to the analog input reflected in the upper tracing. As may be seen, the frequency of integrator marks is proportional to the area under the lower half of the input tracing.

To insure proper operation, the integrators should be adjusted as follows: With a \pm 10 - $V$ zero-center bias meter attached, the gate terminals shorted, R1 adjusted for maximum sensitivity, and the input device connected, adjust R3 until the meter reads zero with no input signal.

With the unit operating in the log mode, the maximum deviation of the input/output function from a true log function is approximately $\pm 5 \%$, as

\section{REFERENCES}

BITTERMAN, M. E. Animal learning. In J. B. Sidowski (Ed), Experimental methods and instrumentation in psychology. New York: McGraw-Hill, 1966. Chap. 11.

KOOTSEY, J. M. FET and IC keep oscillator linear. Electronics, 1968, 41, 84.

*Development of this apparatus was supported by Grant MH17736 from the Public Health Service. 